\title{
Global electron content: a new conception to track solar activity
}

\author{
E. L. Afraimovich ${ }^{1}$, E. I. Astafyeva ${ }^{1}$, A. V. Oinats ${ }^{1}$, Yu. V. Yasukevich ${ }^{1}$, and I. V. Zhivetiev ${ }^{2}$ \\ ${ }^{1}$ Institute Solar-Terrestrial Physics SB RAS, Irkutsk, Russia \\ ${ }^{2}$ Institute of Cosmophysical Research and Radiowave Propagation, FEB RAS, Petropavlovsk-Kamchatskii, Russia
}

Received: 19 January 2007 - Revised: 23 July 2007 - Accepted: 23 July 2007 - Published: 26 February 2008

\begin{abstract}
We developed a method and programs for estimation of the global electron content (GEC) from GPS measurements, using the ionosphere models IRI-2001 and NeQuick. During the 23rd cycle of solar activity, the value of GEC varied from 0.8 to $3.2 \times 10^{32}$ electrons, following changes in the solar extreme ultra violet (EUV) radiation and solar radio emission at $10.7-\mathrm{cm}$ wavelength. We found a strong resemblance of these variations, with discernible 11-year and 27-day periodicities. A saturation effect of GEC is found when F10.7 increases. We found that GEC is characterized by strong seasonal (semiannual) variations with maximum relative amplitude at about $10 \%$ during the rising and falling parts of the solar activity and up to $30 \%$ during the period of maximum. It was found that the relative difference between model and experimental GEC series increase as the smoothing time window decreases. We found that GEC-IRI seasonal variations are out-of-phase with experimental GEC values. The lag between model and experimental maximum of GEC values can reach several tens of days. The variations of GEC lag, on average, 2 days after those of F10.7 and UV. GEC completely reflects the dynamics of the active regions on the solar surface. The amplitude of the 27-day GEC variations decreases from $8 \%$ at the rising and falling solar activity to $2 \%$ at the maximum and at the minimum. We also found that the lifetime of contrast long-living active formations on the Sun's surface in EUV range for more than 1 month exceeds the one in radio range $(10.7 \mathrm{~cm})$.
\end{abstract}

Keywords. Ionosphere (Solar radiation and cosmic ray effects; Instruments and techniques) - Radio science (Ionospheric physics)

Correspondence to: E. I. Astafyeva

(elliada@iszf.irk.ru)

\section{Introduction}

The Earth's ionosphere is an important part of the near space environment; its condition is determined by the solar radiation flux within the different wavelength ranges (IvanovKholodny and Nikolsky, 1969; Akasofu and Chapmen, 1972; Krinberg and Taschilin, 1984). Many works have been devoted to the study of the ionosphere variability due to solar activity changes. Such studies are of great importance for the ionosphere modeling because a periodicity in the intensity of UV solar irradiance is clearly seen in long data records (Kane et al., 1995). To reproduce these variations, ionosphere models rely on solar indices. Ideally it should be an index that tracks the solar cycle changes in the EUV wavelength range, since this part of the solar spectrum affects the ionosphere (Bilitza, 2001). However, such indices cannot be observed at the ground and are only available for the relatively short time periods covered by satellite UV instruments. Thus, most ionospheric modelers use the sunspot number (number of dark spots on the solar disc) and the solar radio flux at $10.7 \mathrm{~cm}$ wavelength (F10.7) as solar indices, since both can be observed from the ground and long data records exist.

On the other hand, the reconstruction of solar radiation parameters from ionosphere observational data is of great interest. After the first paper (Beynon and Brown, 1959), many attempts were undertaken to reveal solar flux characteristics from ionosphere data (Nusinov, 2004). A necessity of the problem still exists, in spite of the development of modern extra-atmospheric (satellite) facilities for solar radiation detection. However, the local features of the ionosphere parameters make it difficult for the estimation of quantitative solar radiation characteristics from ionospheric data.

Quasi 27-day variations caused by solar rotation are one of the main factors of solar radiation influence on the ionosphere state. Many works have been devoted to researching of this factor (Akasofu and Chapmen, 1972;

Published by Copernicus Publications on behalf of the European Geosciences Union. 
Ivanov-Kholodny and Nikolsky, 1969; Vitinsky et al., 1986; Jakowski et al., 1991, 1998, 2002). However, quasi 27-day modulation of local ionosphere parameters can be masked by many other factors. The latter makes it impossible to derive reliable numerical characteristics of the 27-day variations of the solar ultraviolet radiation.

A new approach for studying and gaining a better understanding of the Sun-Earth connections was proposed, for the first time, by Afraimovich et al. (2006a). This approach is concerned with determining the global electron content (GEC) that is equal to the total number of electrons in the near-Earth space environment within the GPS orbital altitude of about $20200 \mathrm{~km}$. The main advantage of this approach is in the disappearance of local features of the ionosphere parameters and in determining the dynamics of global characteristics.

The objectives of this paper are to study the dynamics of the global electron content during the 23rd cycle of solar activity and to compare it with changes in the solar EUV radiation in the $0.1-50 \mathrm{~nm}$ wavelength range monitored by the Solar EUV Monitor spectrometer aboard the Solar Heliospheric Observatory (SOHO) (Judge et al., 1998; data in units of $10^{11}$ photons $\mathrm{cm}^{-2} \mathrm{~s}^{-1}$ are available from http://www.usc.edu/dept/space_science/semdatafolder/ long/); daily values of sunspot number Rsn (http://www. spacewx.com) and the 10.7 index which is equal to the solar radiation flux on the wavelength of $10.7 \mathrm{~cm}$ in s.f.u. units $\left(10^{-22} \mathrm{~W} \mathrm{~m}^{-2} \mathrm{~Hz}^{-1}\right)$; http://www.drao-ofr.hia-iha.nrc-cnrc. gc.ca/icarus/www/current.txt. We also compare observational GEC data with IRI-2001 and NeQuick model GEC values.

\section{The method of global electron content calculation}

Our method is based on the use of global ionosphere maps (GIM) of total electron content (TEC) generated based on the data from the International GPS receivers network by several laboratories. GIM are available from the Internet site ftp:// cddisa.gsfc.nasa.gov/pub/gps/products/ionex/; GIM contain data of the vertical TEC with a spatial step of $5^{\circ}$ of longitude and $2.5^{\circ}$ of latitude - the elementary GIM cell, and temporal step of $2 \mathrm{~h}$. The total number of cells equals 5184 (72 cells along the longitude multiplied by 72 cells along the latitude).

Global electron content $\mathrm{G}(\mathrm{t})$ is calculated by the summation of the absolute vertical TEC values $\mathrm{I}_{i, j}$ multiplied by the cell's area $S_{i, j}$ over all GIM cells (Afraimovich et al., 2006a, b):

$$
\begin{aligned}
G & =\sum_{i, j} I_{i, j} \cdot S_{i, j} \\
& =\sum_{i, j} I_{i, j} \cdot R_{E}^{2} \cdot \Delta \varphi \cdot\left[\sin \theta_{j}-\sin \left(\theta_{j}+\Delta \theta\right)\right],
\end{aligned}
$$

where $i, j$ are the indices of the GIM cell, $\varphi_{j}$ and $\theta_{j}-$ longitude and latitude of the GIM cell with angular scales along longitude and latitude equal to $\Delta \varphi$ and $\Delta \theta$, accordingly, $R_{E}$ - the Earth's radius. The absolute vertical TEC value is defined by the well-known formula $I_{i, j}=\int_{R_{E}}^{R_{E}+h_{\max }} N_{i, j}(r) d r$, where $N_{i, j}(r)$ is a vertical electron density profile in the GIM cell, $r$ - the radial coordinate, $h_{\max }$ - the upper height of integration.

We suggest a unit of GEC measurement GECU that is equal to $10^{32}$ electrons. GEC estimation accuracy is higher than TEC determination accuracy for each GIM cell (about 10-20\% - Mannucci et al., 1998; Schaer et al., 1998), when averaging independent TEC values for the entire globe. This allows us to reveal GEC variations and trends of considerable amplitude, which is impossible in the case of TEC analysis in the separated GIM cell or GPS site. In our work we used GIM for the period of 1996-2006.

A comparative study showed that the results of GEC estimation slightly depend on data of a certain laboratory. Hereinafter, all calculations are carried out for JPLG data (Mannucci et al., 1998). The total number of calculated GEC values for 3868 days with two-hour time resolution (from Day 152,1998 ) is $3868 \times 12=46416$. For time period from 1 January 1996 to Day 152, 1998, we used the daily mean values of TEC, which are available from ftp://ftp.unibe.ch/aiub/ CODE/.

We smoothed the $G(t)$ series with the time window of 1 day, so diurnal variations appear to be averaged and, therefore, effects of quick GEC changes (such as geomagnetic disturbances, solar flares, etc.) cannot be distinguished, since these processes are the topics of a special study.

Estimation of the number of electrons for each GIM cell (one term $g_{i, j}=I_{i, j} \cdot S_{i, j}$ in Eq. 1) is carried out within a part of a constant cross-sectional area, i.e. spherical divergence is not taken into account. This leads to the appearance of hard errors in the estimations of the total number of electrons by Eq. (1). The theoretical number of electrons within the spherical layer from height $R_{E}$ to height $R_{E}+h_{\max }$ can be represented by an integral of the general electron density function $N(r, \theta, \varphi)$

$$
G_{\text {teor }}=\int_{R_{E}}^{R_{E}+h_{\max }} \int_{-\pi / 2}^{\pi / 2} \int_{-\pi}^{\pi} N(r, \theta, \varphi) r^{2} \cos \theta d r d \theta d \varphi .
$$

With the assumption of the spherically symmetrical ionosphere over a single GIM cell, or, in other words, the electron density profile does not depend on angular coordinates $\theta$ and $\varphi$ within a single GIM cell, expression (2) for the GIM cell with the indices $i, j$ can be reduced to

$$
g_{i, j \text { teor }}=\Delta \varphi \cdot\left[\sin \theta_{j}-\sin \left(\theta_{j}+\Delta \theta\right)\right] \int_{R_{E}}^{R_{E}+h_{\max }} N_{i, j}(r) r^{2} d r .
$$

As one can see, Eq. (3) contains factor $r^{2}$ under the sign of integration. So it is obvious that it is not possible to estimate 
precisely the number of electrons without knowledge of the vertical electron density profile, as we have only vertical TEC above a GIM cell on the Earth's surface.

Taking into account Eq. (3), the relative error of GEC estimation in Eq. (1) for each cell can be found from the expression:

$$
\begin{aligned}
\varepsilon & =\frac{g_{i, j \text { teor }}-g_{i, j}}{g_{i, j}} \times 100 \% \\
& =\frac{\int_{R_{E}}^{R_{E}+h_{\max }} N_{i, j}(r)\left(r^{2}-R_{E}^{2}\right) d r}{R_{E}^{2} \cdot \int_{R_{E}}^{R_{E}+h_{\max }} N_{i, j}(r) d r} \times 100 \% .
\end{aligned}
$$

Figure 1 shows the relative error $\varepsilon, \%$ (solid lines), depending on the upper height of the integration $h_{\max }$, calculated using Eq. (4). We use NeQuick model profile formulation (Leitinger et al., 2005) for the electron density profile $N_{i, j}(r)$ (dotted lines) calculation, since the NeQuick model produces a more realistic topside ionosphere against IRI-2001 (Coisson and Radicella, 2005, 2004; Coisson et al., 2002). The lines correspond to different values of parameter $\boldsymbol{k}$ from the model NeQuick, which characterizes the rapidity of the decreasing electron density above the maximum of the F2 layer. The high $\boldsymbol{k}$ values correspond to the low rapidity of the decreasing electron density (on the Fig. 1: black line $-k=2$; dark gray $-k=5$ and light gray $-k=8$ ).

As one can see from Fig. 1, the relative errors increase as the upper height of the integration $h_{\max }$ increases up to $\sim 2000 \mathrm{~km}$. Then, the growth of the error values becomes slower and relative errors tend to a limit of about $10-20 \%$, because the main number of electrons is located at ionospheric heights from 90 to $1500 \mathrm{~km}$.

Thus, the corresponding systematic GEC correction is about $10-15 \%$. However, this factor influences mainly the absolute value of GEC, but not on the form or character of the GEC variations, according to Eq. (1).

Here we use the term global electron content since GIM are presented for the entire globe. At the same time TEC estimations near the geographical poles are very rough in GIM. For these regions the TEC maps of the North Pole (NOR) presented at the site http://www.kn.nz.dlr.de/daily/tec-np/are more representative. However, the contribution of high latitude regions to GEC is essentially smaller than the one of middle and low latitudes. Thus, as the first approximation, the $\mathrm{G}(\mathrm{t})$ value calculated by Eq. (1) is sufficiently close to the total number of electrons around the Earth.

\section{Modeling of GEC by the International Reference Ionosphere 2001 (IRI-2001) and by NeQuick models}

For global electron content modeling we should use an ionosphere model which satisfies at least two conditions: (1) it must reproduce an electron density profile not only up to the

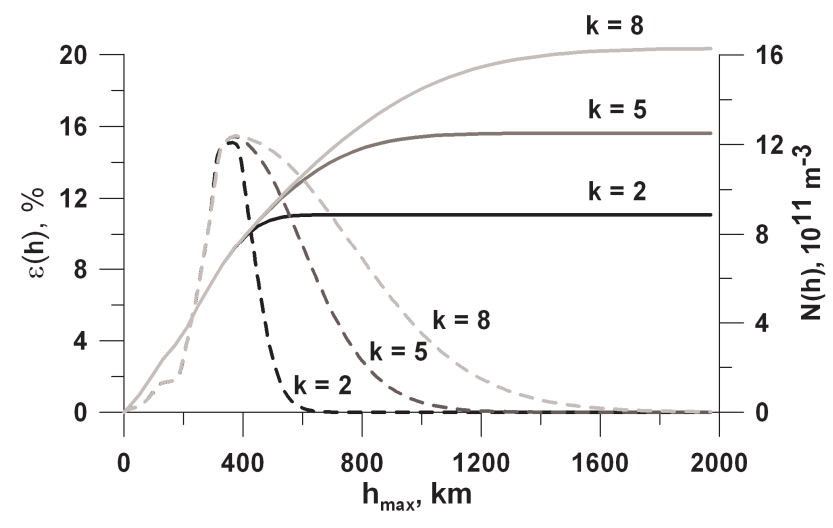

Fig. 1. Relative error of GEC estimation, $\varepsilon, \%$ (solid lines) depending on the upper height of integration $h_{\max }$. The profile of electron concentration $\mathrm{N}(\mathrm{h})$ calculated by the NeQuick model is shown by the dotted lines. Different lines correspond to different values of parameter $k$ which characterizes the rapidity of the decrease in electron density above the maximum of F2 layer (black line $-k=2$; dark gray $-k=5$ and light gray $-k=8$ ).

maximum of the F2 layer, but also beyond the maximum; (2) it must reproduce TEC values or electron density profile at any point on the Earth. Lately, several ionosphere models have been developed, such as IRI (Bilitza, 1990 and 2001; http://nssdc.gsfc.nasa.gov/space/model/ionos/iri.html) and NeQuick (Giovanni and Radicella, 1990; Radicella and Leitinger, 2001; Leitinger et al., 2005; Coisson et al., 2002, 2005; http://www.itu.int/ITU-R/software/studygroups/rsg3/ databanks/ionosph/Rec531/), which are recommended by the International Telecommunication Union ITU-R (ITU2004), as suitable methods for TEC estimations.

The NeQuick model is based on the analytical model of the electron density profile originally proposed by G. Di Giovanni and S. M. Radicella; the DGR model (Giovanni and Radicella, 1990) produces a more realistic topside profile shape. However, the NeQuick version available as a free download can calculate the monthly median profile for each location on the Earth (and accordingly only month median TEC value).

Using the IRI 2001 we calculated modeled GEC M(t) in two stages. First, IRI TEC values were calculated throughout the globe in latitude-longitude mesh points with a step of $5^{\circ}$ in longitude and $2.5^{\circ}$ in latitude during all time steps of $2 \mathrm{~h}$. Second, derived TEC values were integrated using the technique described earlier for GIM (Eq. 1). GEC-NeQuick values $\mathrm{N}(\mathrm{t})$ were calculated in a similar way as $\mathrm{M}(\mathrm{t})$ values. But because the NeQuick produces "monthly" TEC data (same $24 \mathrm{~h}$ dependence for every day of a month), we smoothed the dependence $\mathrm{N}(\mathrm{t})$ with a time window of 81 days to obtain the curve in Fig. 2c.

The main parameter in the case of the TEC calculation is the upper height for the electron density profile integration $h_{\max }$. Calculations of GEC-IRI for the upper height 


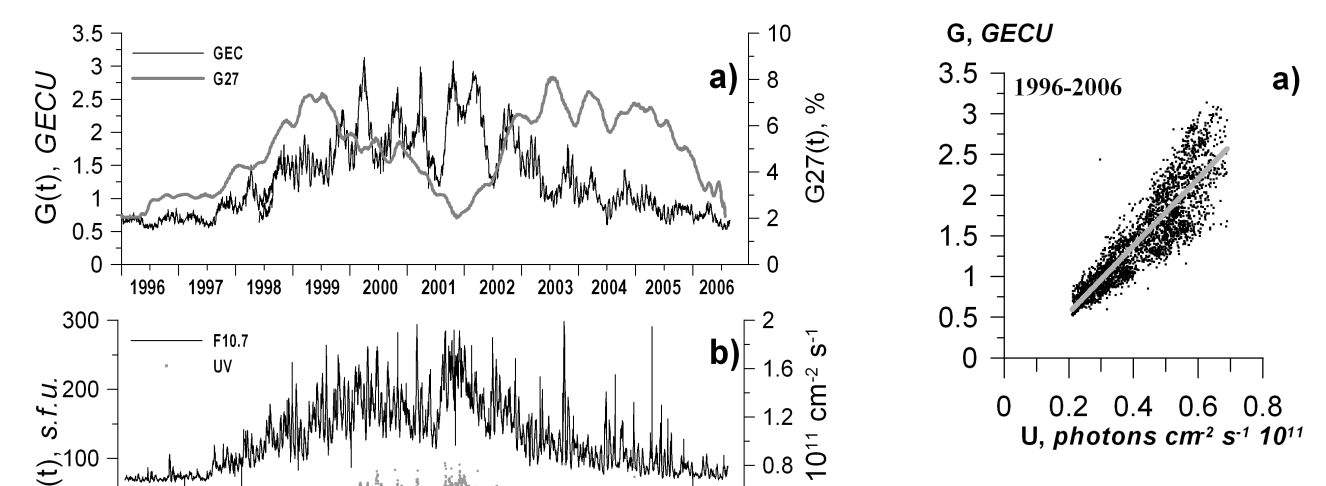

G, GECU
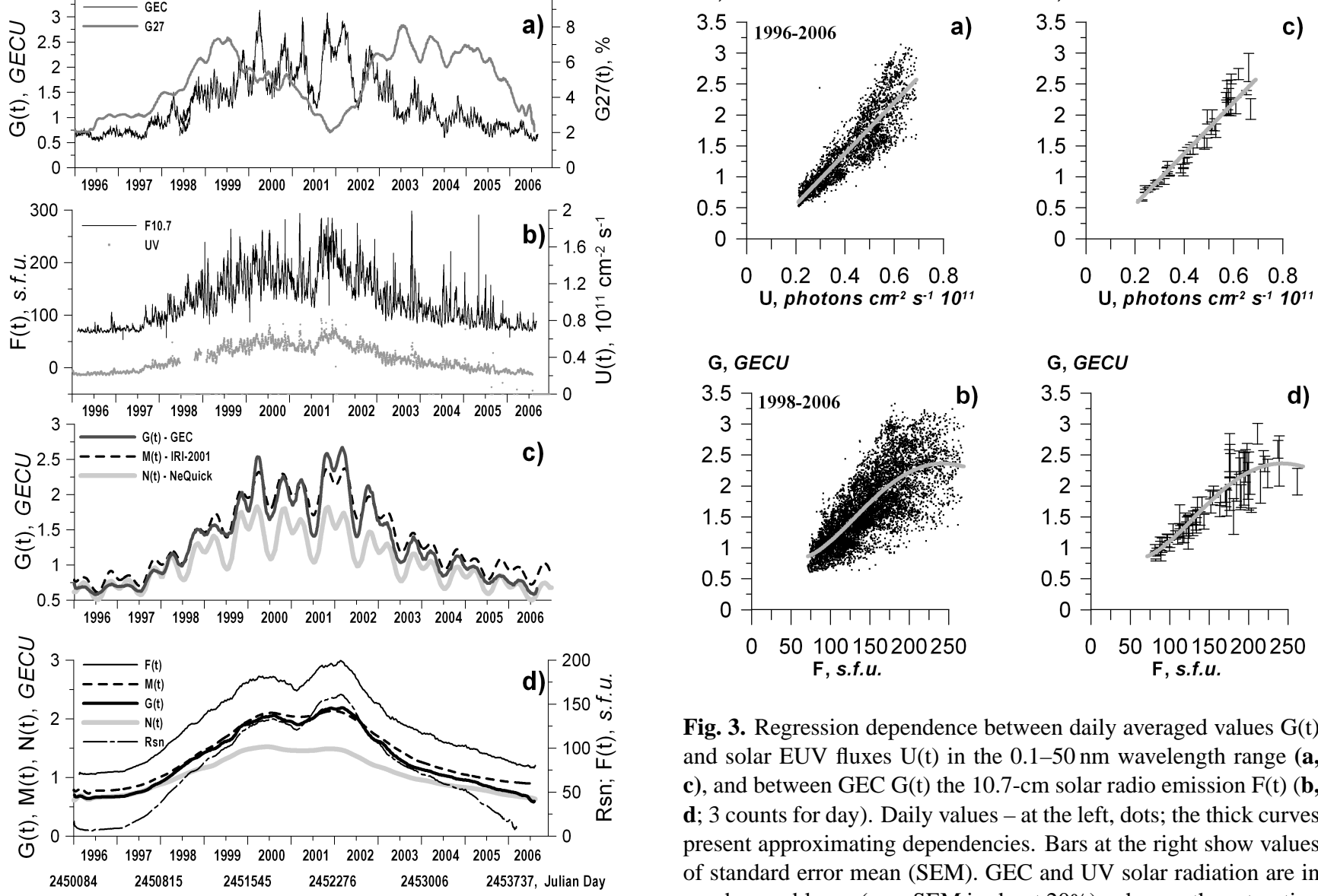

Fig. 3. Regression dependence between daily averaged values $\mathrm{G}(\mathrm{t})$ and solar EUV fluxes $\mathrm{U}(\mathrm{t})$ in the $0.1-50 \mathrm{~nm}$ wavelength range (a, c), and between GEC G(t) the 10.7-cm solar radio emission $\mathrm{F}(\mathrm{t})(\mathbf{b}$, d; 3 counts for day). Daily values - at the left, dots; the thick curves present approximating dependencies. Bars at the right show values of standard error mean (SEM). GEC and UV solar radiation are in good resemblance (a, c; SEM is about $20 \%$ ), whereas the saturation effect is obvious of GEC as F10.7 increases (b, d; SEM is about

Fig. 2. (a) GEC variations $\mathrm{G}(\mathrm{t})$ for the whole globe (black thick curve), relative amplitude (\%) of 27-day variations of $\mathrm{G}(\mathrm{t})$, filtered in the time period range of 20-40 days (gray curve); (b) the 10.7$\mathrm{cm}$ solar radio emission $\mathrm{F}(\mathrm{t})$ (black) and solar EUV fluxes $\mathrm{U}(\mathrm{t})$ in the $0.1-50 \mathrm{~nm}$ wavelength range (gray); (c) experimental GEC G(t) and modeled GEC using the IRI-2001 M(t) and the NeQuick N(t), smoothed with a 81-day time window; (d) smoothed with the 365day time window dependencies of $F(t), M(t), G(t), N(t)$ and daily sunspot numbers Rsn.

$h_{\max }=20000 \mathrm{~km}$, as expected (Coisson and Radicella, 2004, 2005; Coisson et al., 2005), showed a significant overestimation of GEC values (more than 6 times). This overestimation is related to the fact that the IRI electron density profile is close to the real ionosphere profile only up to the height of $1500 \mathrm{~km}$ and it becomes almost constant above $1500 \mathrm{~km}$. We found a minimal absolute difference between the experimental and IRI modeling GEC dependencies for $h_{\max }=2000 \mathrm{~km}$ (Afraimovich et al., 2006b, c). Therefore, all GEC-IRI estimations have been performed for $h_{\max }=2000 \mathrm{~km}$. As for GEC-NeQuick, all calculations were performed for $h_{\max }=20000 \mathrm{~km}$. $50 \%)$.

\section{Experimental and modeling GEC during the 23rd cy- cle of solar activity}

The GEC value $\mathrm{G}(\mathrm{t})$ during the 23rd cycle of solar activity varied from 0.8 to 3.5 GECU (Fig. 2a, black curve). When comparing the GEC series with the solar activity index F10.7 (Fig. 2b, black line) and with variations of solar UV radiation (Fig. 2b, dots) one can notice a good agreement between these variations. The 11 -year periodicity is very perceptible in the data series of $G(t)$ (black thick curve), $F(t)$ (black thin curve) and the daily sunspot number Rsn (dash-dot curve) smoothed by a one-year time window (Fig. 2d). One can notice an increasing of the values at the maximum of the solar activity cycle and their decreasing by its minimum.

Figure 3 presents the regression dependences between the daily averaged values $\mathrm{G}(\mathrm{t})$ and the solar EUV radiation $\mathrm{U}(\mathrm{t})$ - $(\mathrm{a}, \mathrm{c})$, and between $\mathrm{G}(\mathrm{t})$ and the 10.7-cm solar radio emission $\mathrm{F}(\mathrm{t})-(\mathrm{b}, \mathrm{d} ; 3$ counts per day). These dependencies are obtained for the $0-250$ s.f.u. F10.7 range and for the $0-0.8 \times 10^{11}$ photons $\mathrm{cm}^{-2} \mathrm{~s}^{-1}$ EUV range. Single F10.7 $(>250)$ and EUV ( $>0.8$ ) spikes caused by strong solar flares 


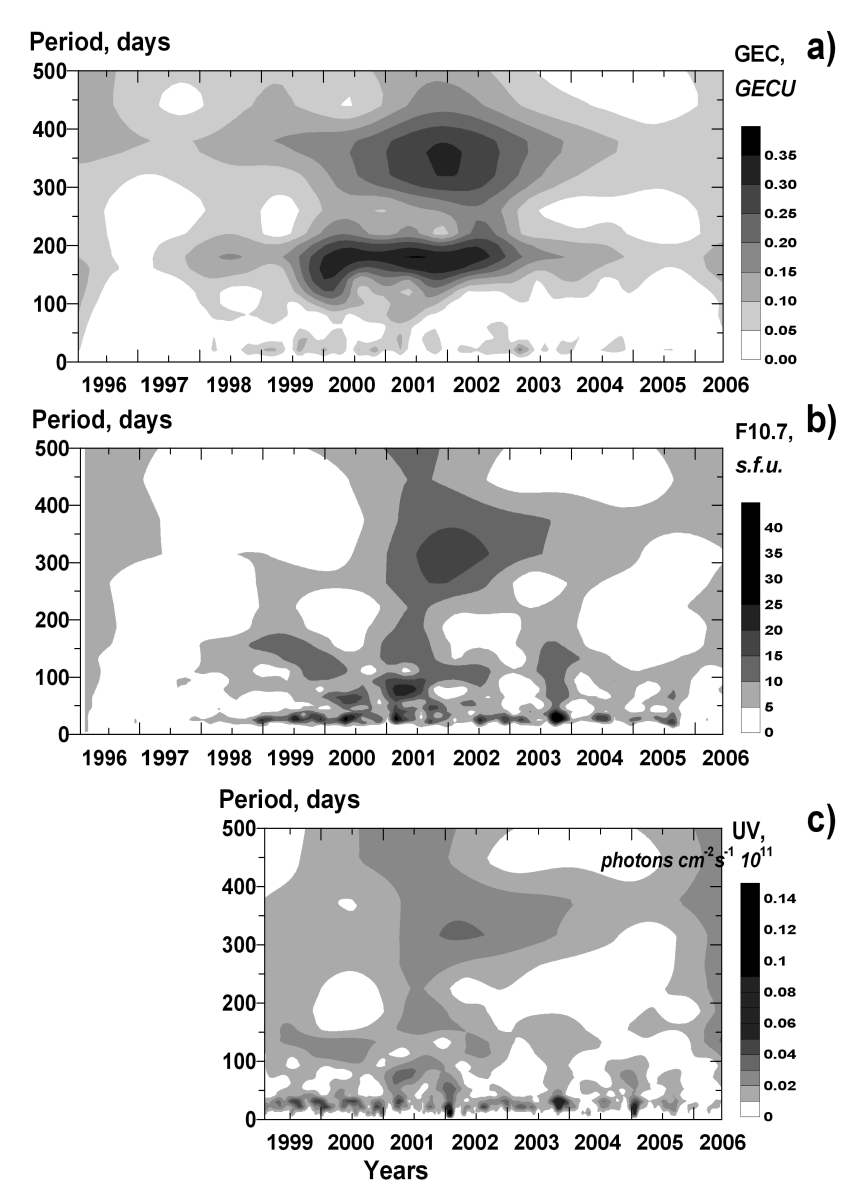

Fig. 4. Amplitude wavelet spectra of GEC (a) and F10.7 (b) and EUV (c) in the time period range of 0-500 days.

are excluded. Thick curves present approximating dependencies; the bars on the right panel show values of the standard error mean (SEM). As one can see, GEC and UV solar radiation have a good resemblance (a, c-SEM is about 20\%) that is in good agreement with the modern empirical models of the ionosphere (Bryunelli and Namgaladze, 1988).

From Figs. 3b, d one can see the saturation effect of GEC when F10.7 increases significantly (SEM is about 50\%). This agrees with the results obtained before by Balan et al. (1996), where data of the ionospheric electron content, peak electron density $(N m F 2)$ and values of solar EUV (50-1050 ̊) fluxes were analysed to study the variations in the ionosphere during the intense solar cycles 21 and 22. It was shown that the ionosphere responds linearly to the solar EUV fluxes, whereas its variations with the conventional solar activity index F10.7 are nonlinear. Liu et al. (2006) examined a long series of daily solar EUV data and $N m \mathrm{~F} 2$ data at 20 ionosonde stations and found the saturation effect of $N m \mathrm{~F} 2$ for high values of both the solar EUV and F10.7 index. However, the saturation effects of $N m \mathrm{~F} 2$ with solar EUV was found to have a latitudinal dependence, and correlation coefficients between $N m \mathrm{~F} 2$ and solar EUV were found to be higher than those of

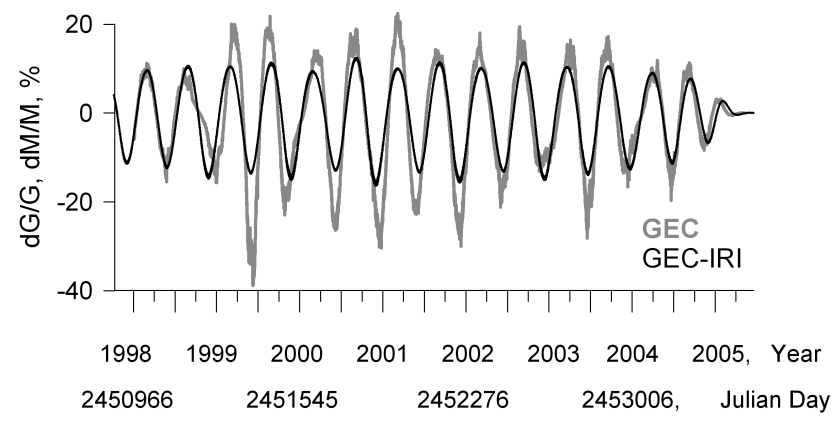

Fig. 5. Relative amplitude $(\%)$ of $\mathrm{G}(\mathrm{t})$ and $\mathrm{M}(\mathrm{t})$ variations filtered in the time period range of 100-300 days.

F10.7. Thus, observations show that the use of F10.7 in ionosphere modeling instead of UV leads to an error in estimation of ionosphere parameters during high solar activity.

We calculated modeled GEC using the IRI-2001 and NeQuick and compared them with experimental GEC values (Fig. 2c). GEC-IRI dependence $M(t)$ is shown by a dash curve. GEC-NeQuick dependence $\mathrm{N}(\mathrm{t})$ is presented by a light gray curve. Comparison between the experimental $G(t)$ and modeled $\mathrm{M}(\mathrm{t})$ and $\mathrm{N}(\mathrm{t})$ series, smoothed by a one-year time window, shows good agreement between the values of $\mathrm{G}(\mathrm{t})$ and $\mathrm{M}(\mathrm{t})$ during the whole period under consideration (Fig. 2d). For the GEC value calculated by NeQuick model one can see good agreement with experimental values during minimums of solar activity cycle but significantly underestimated values of $\mathrm{N}(\mathrm{t})$ during the maximum of solar activity cycle (Fig. 2d, light gray curve).

\section{Semiannual variations of GEC}

We investigated the spectral properties of the GEC, F10.7 and EUV series during the 23rd cycle. Figure 4 shows wavelet amplitude spectra (Kumar et al., 1997) in the time period from 0 to 500 days. As one can see, there are distinct semiannual and annual variations of GEC (panel a), particularly during the high solar active period (2000-2002). As one can expect, these GEC variations are not correlated with F10.7 and EUV variations (panels $\mathrm{b}$ and $\mathrm{c}$ ).

Figure $2 \mathrm{c}$ presents the comparison between experimental GEC G(t) - black curve, and modeled GEC, using the IRI$2001 \mathrm{M}(\mathrm{t})$ and the NeQuick $\mathrm{N}(\mathrm{t})$, smoothed with a 81-day time window (dash and gray curves). Figure 5 illustrates a comparison between the variations of the relative amplitudes $\mathrm{dG}(\mathrm{t}) / \mathrm{G}(\mathrm{t}), \%$ (gray curve) and $\mathrm{dM}(\mathrm{t}) / \mathrm{M}(\mathrm{t}), \%$ (black curve), filtered in the time period range of 100-300 days. We found that GEC is characterized by strong seasonal (semiannual) variations with a maximum relative amplitude of about $10 \%$ during the rising and falling parts of the solar activity period and up to $30 \%$ during the period of maximum (Fig. 5). 


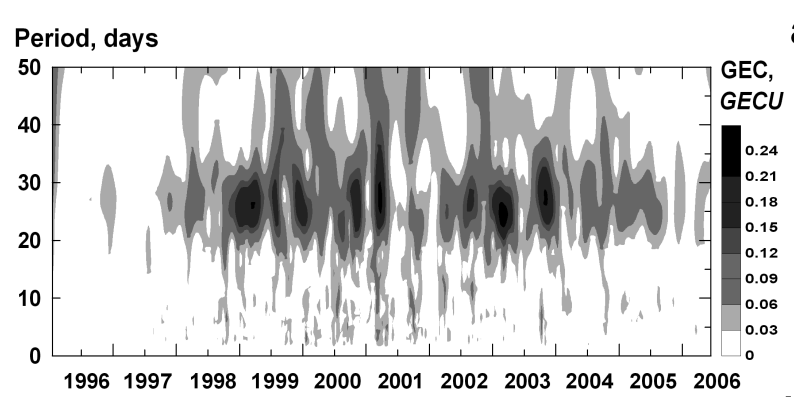

Period, days

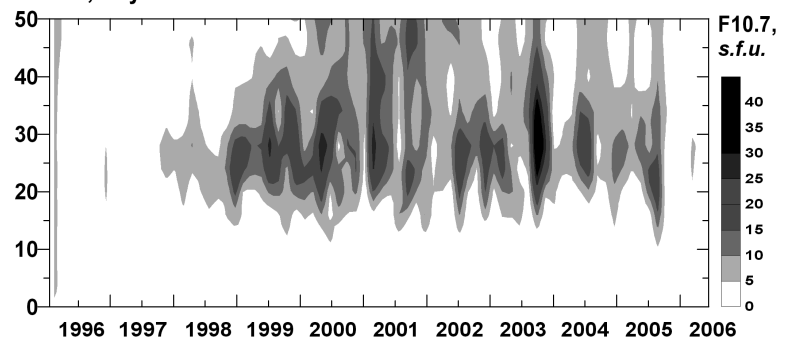

Period, days

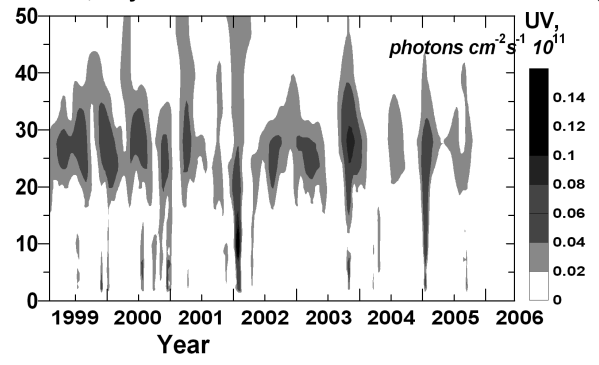

Fig. 6. Amplitude wavelet spectra of GEC (a) and F10.7 (b) and EUV (c) in the time period range of 0-50 days.

This fact agrees well with the results from studying the mean TEC dependence over Japan during 2000-2003 (Oyama et al., 2005). This also is in agreement with the fact that neutral atmosphere density reaches its maximum values in April and October (Krinberg and Taschilin, 1984).

We found that GEC-IRI seasonal variations are out-ofphase with experimental GEC values (Fig. 5). The lag between model and experimental maximum GEC values can reach several tens of days.

\section{27-day variations of GEC - comparison with varia- tions of solar UV and radio emission}

As it was mentioned above, GEC has 27-day variations which are related to the Sun's rotation. Wavelet amplitude spectra of GEC in the time period from 0 to 50 days shows 27-day variations (Fig. 6a) similar to F10.7 and EUV variations (panels b and c). 27-day variations related to the Sun's rotation are obvious in the data series of $\mathrm{G}(\mathrm{t}), \mathrm{F}(\mathrm{t})$ and $\mathrm{U}(\mathrm{t})$, as one can see from Figs. 2a, b. Correlation analysis for all data from 1996 to 2006 displays a high similarity between

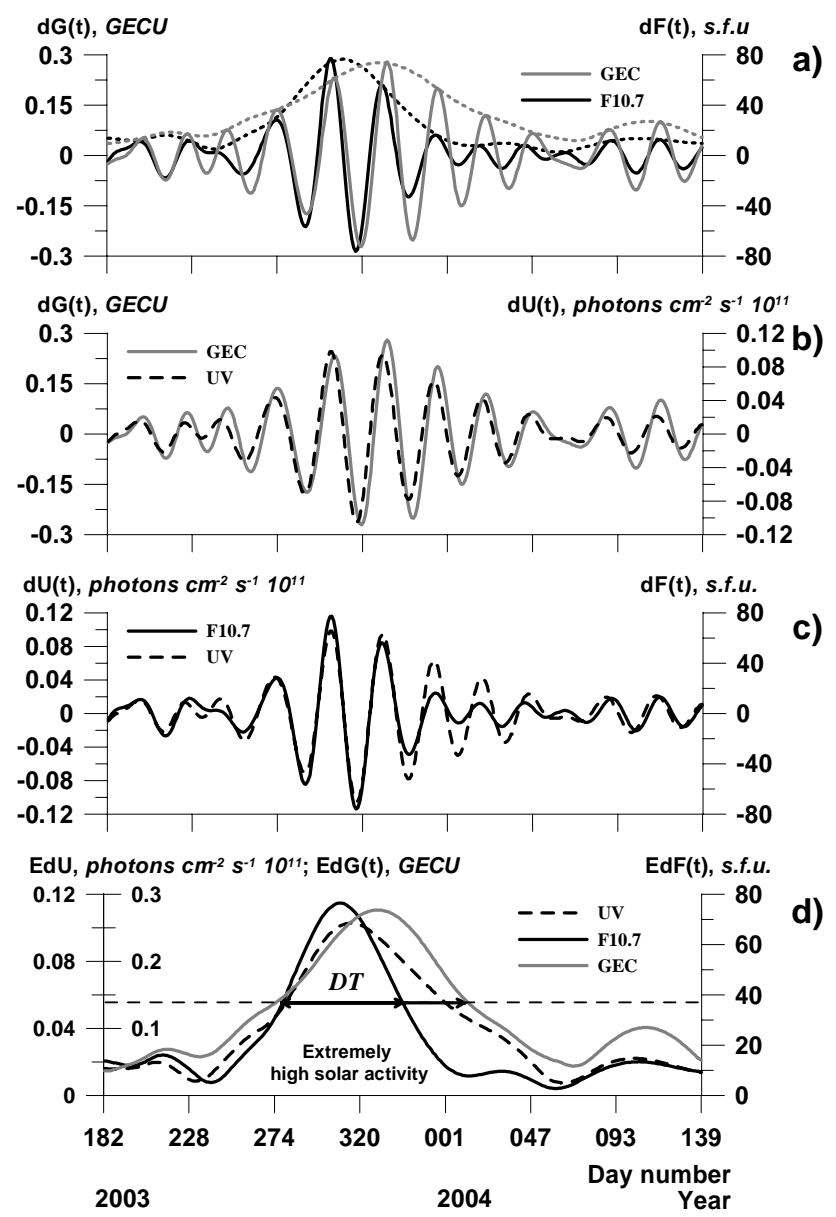

Fig. 7. The 27-day variations of $d G(t), d F(t)$ and $d U(t)$ for 20032004 years $(\mathbf{a}, \mathbf{b}, \mathbf{c})$; (d) the same, but for the envelopes $\mathrm{EdG}(\mathrm{t})$, $\operatorname{EdF}(\mathrm{t})$ and $\operatorname{EdU}(\mathrm{t})$, corresponding to the 27-day variations of the $\mathrm{dG}(\mathrm{t}), \mathrm{dF}(\mathrm{t})$ and $\mathrm{dU}(\mathrm{t})$.

the 27-day variations of the $\mathrm{dG}(\mathrm{t}), \mathrm{dF}(\mathrm{t})$ and $\mathrm{dU}(\mathrm{t})$, filtered from the initial series $\mathrm{G}(\mathrm{t}), \mathrm{F}(\mathrm{t}), \mathrm{U}(\mathrm{t})$ within the time period range of 20-40 days and smoothed with the 1-day time window (see below).

Figures 7a, b, c show 27-day variations of the $\mathrm{dG}(\mathrm{t})$ - gray curve, $\mathrm{dF}(\mathrm{t})$ - black curve, and $\mathrm{dU}(\mathrm{t})$ - dash curve, for the time interval from 1 July 2003 to 18 May 2004. This period was selected because of the extremely high solar activity in October-November 2003 (Veselovsky et al., 2004). GEC variations were calculated for each day within the interval from 17:00 to 21:00 UT (the same way as for F10.7 data).

One can see that the 27-day variations of $\mathrm{dF}(\mathrm{t})$ and $\mathrm{dU}(\mathrm{t})$ are in-phase (Fig. 7c), whereas 27-day GEC variations lag, on average, for 2 days after variations in solar $10.7 \mathrm{~cm}$ radio emission and solar UV radiation (Figs. 7a and b). One can draw the same conclusion while looking at the correlation functions for $\mathrm{G}(\mathrm{t}), \mathrm{F}(\mathrm{t})$ and $\mathrm{U}(\mathrm{t})-$ Fig. 8 .

Correlation analysis of the data for the period from 1999 to 2006 (102 rotations of the Sun) showed a high resemblance 
between 27-day variations of GEC, F10.7 and solar UV (Fig. 8a). The maximum correlation coefficient $\rho_{\max }$ between the data series of GEC and UV reaches 0.94 , between GEC and F10.7 and between F10.7 and EUV is about 0.8 . The mean value of lag $\tau$ between the 27-day variations of GEC and UV radiation estimated from the bar charts is 2.2 days with RMS equal to 1.41 days (Fig. 8d), for GEC and F10.7 - 1.76 days with RMS equal to 2.5 days (Fig. 8c). This can be caused by the systematical forestalling of the UV 27day variations to the 27-day variations of the F10.7 index for 0.46 days, on average, with RMS equal to 2.2 days (Fig. 8b). As a consequence, the scattering of the lag between the data series of GEC and F10.7 (Fig. 8c) significantly exceeds the scattering of the lag between GEC and solar UV radiation (Fig. 8d)

This lag (of about 2 days) of the 27-day GEC variations, as compared with the corresponding changes in the EUV and radio 10.7-cm solar emissions (Figs. 7 and 8), is in agreement with the data presented by Jakowski et al. (1991, 1998, 2002).

Figure $7 d$ shows the envelopes $\operatorname{EdG}(t), E d F(t)$ and $E d U(t)$ of the maximal values of the 27-day variations of the $\mathrm{dG}(\mathrm{t})$, $d F(t)$ and $d U(t)$ presented in Figs. 7a, b, c (these envelopes are also presented in panel a by dotted curves). The horizontal dash line in Fig. 7d indicates a 0.5 level of the envelope of the 27-day variations; the arrows show the time intervals DT, for which the envelope value exceeds 0.5 of its maximal value. The DT value can be used for the estimation of lifetime of contrasting, long-living, powerful, active formations on the Sun's surface which radiate in a different range of wavelengths (Vitinsky et al., 1986; Mordvinov and Willson, 2003; Veselovsky et al., 2004).

One can see from Fig. $7 \mathrm{~d}$ that the increasing of the intensity of the 27-day variations of F10.7, EUV and GEC at the 0.5-level, as compared to the maximum level, occurred almost simultaneously, but with a difference of several days. The intensity of the $G(t)$ and $U(t)$ variations decreased simultaneously, as well. But the intensity of the 27-day variations of $F(t)$ decreases 1 month prior to the intensity of the 27-day variations of UV and GEC starts to decrease. This means that the contrasting, long-living, powerful, active formations on the Sun's surface in the UV range existed during nearly 3 rotations of the Sun, whereas such formations in the F10.7 cm radio range existed during only 2 rotations of the Sun (see also Sect. 7). These facts might be connected to the differences in the origin and spatio-temporal properties of the UV solar radiation and F10.7 solar radio emission in solar active formations (Nusinov and Katyushina, 1994).

We estimated the amplitude of the 27-day variations during the $23 \mathrm{rd}$ solar cycle. Figure $2 \mathrm{a}$ presents the envelope of the relative amplitude of the 27-day variations $\mathrm{G} 27(\mathrm{t})=\mathrm{dG}(\mathrm{t}) / \mathrm{G}(\mathrm{t})$ - gray curve. We squared the dependences $\mathrm{dG} / \mathrm{G}(\mathrm{t})$ and smoothed them by a 365-day time window. It is seen that the maximal deviation of the relative amplitude $\mathrm{G} 27(\mathrm{t})$ decreases from $8 \%$ at the rising and falling

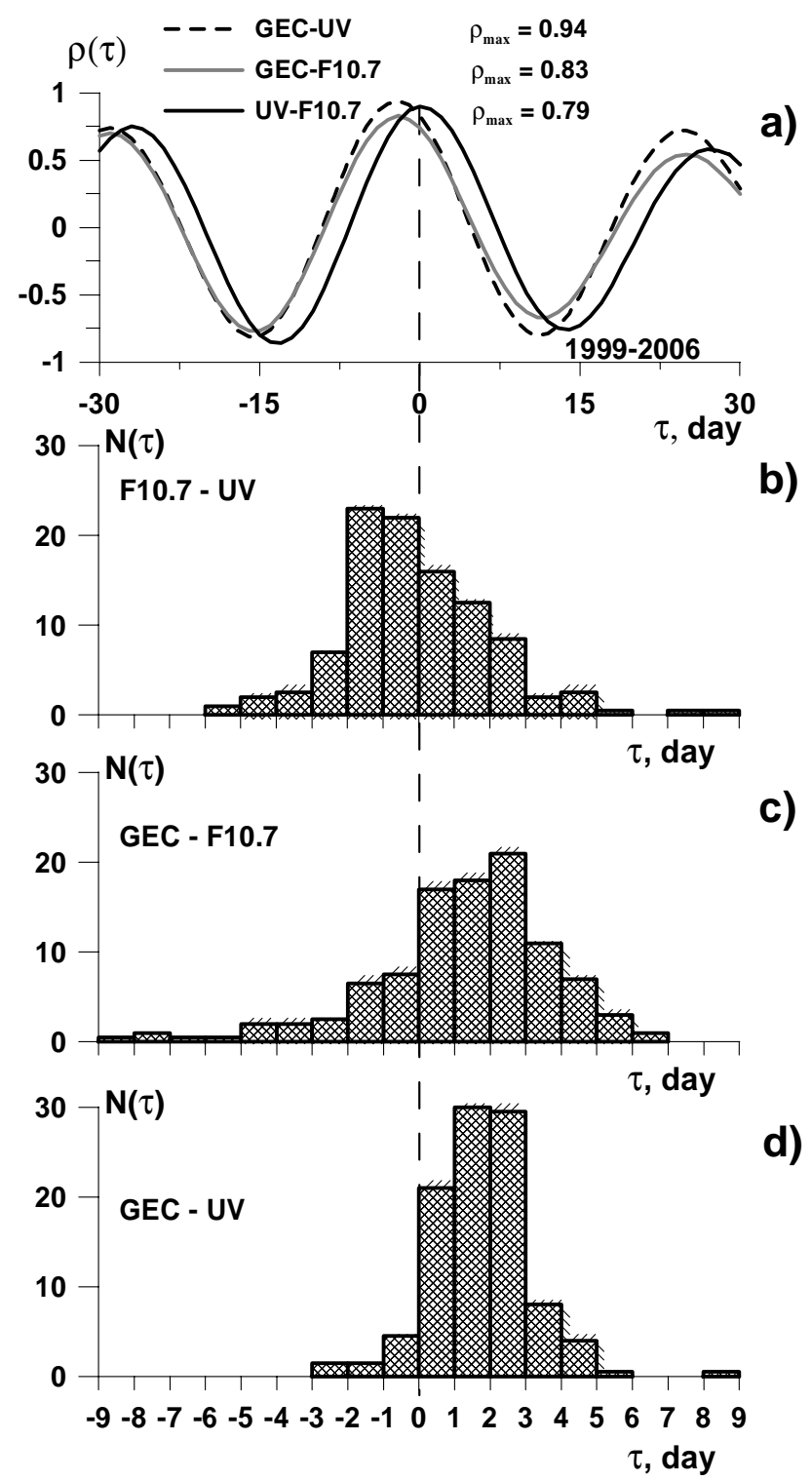

Fig. 8. Correlation functions between the 27-day variations of GEC, index F10.7 and solar EUV for 102 rotations of the Sun. The 27-day variations of the GEC lag for about 2 days after the ones of F10.7 and UV, but RMS of GEC-UV lag is essentially less than the RMS of GEC-F10.7 lag.

solar activity to $2 \%$ at the maximum and at the minimum. The least level of the 27-day modulation is observed at the period when the sunspot number Rsn is maximum or minimum (Fig. 2d). It should be taken into account that smoothing with a 1-year time window causes the real value of the amplitude of 27-day variations to be underestimated in some moments of time. Thus, for the extremely high solar activity in October-November 2003, the maximal deviations of the amplitude of GEC 27-day variations exceeded 0.3 GECU while the mean value of $\mathrm{G}(\mathrm{t})$ was 1 GECU (see Figs. 2a and $7 \mathrm{a}$ ), which is more than $30 \%$. 

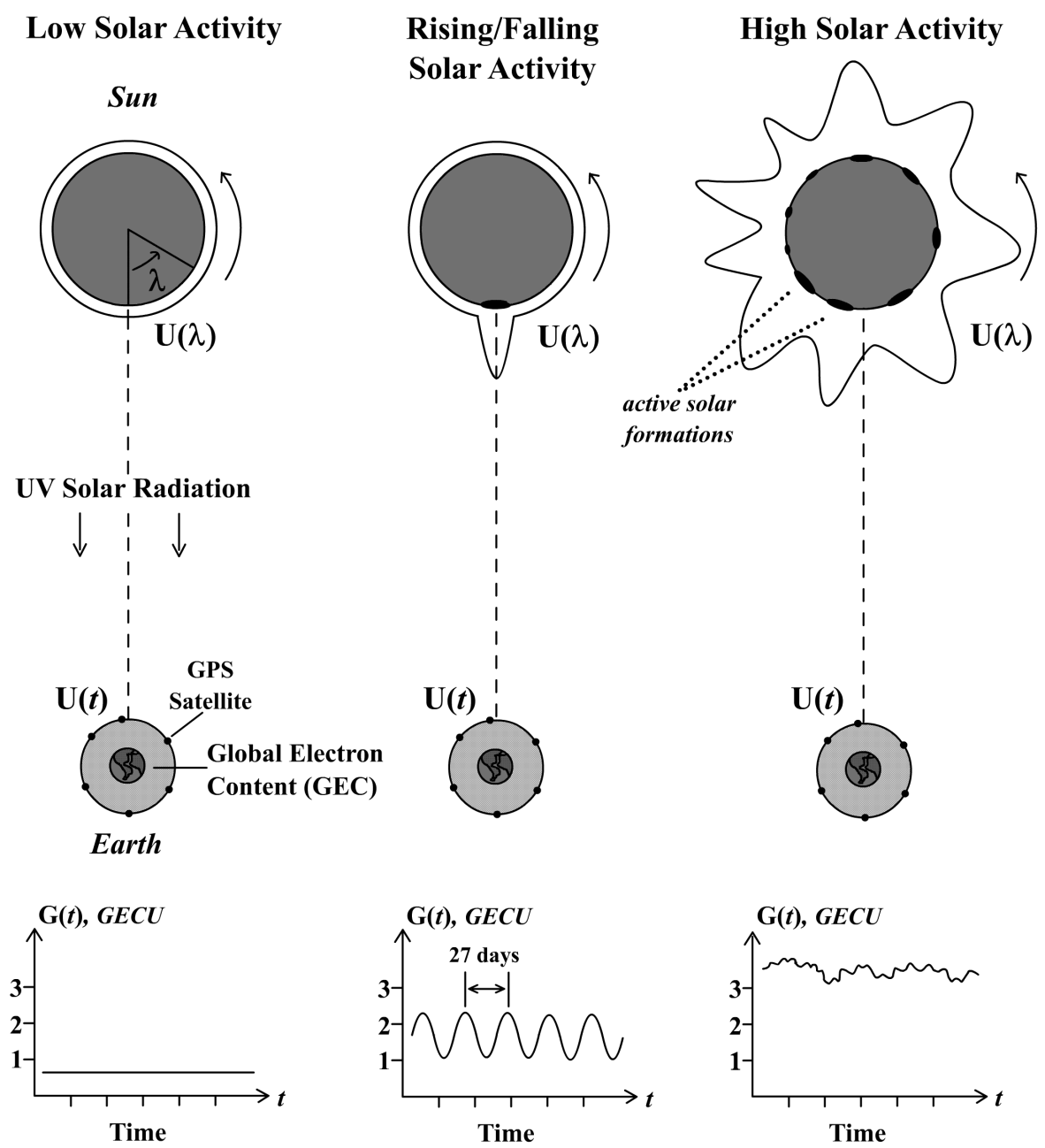

Fig. 9. The illustration of the 27-day GEC variations during solar activity cycle. The pattern inside the GPS orbit shows the cross section of the total volume for which GEC has been calculated.

\section{Discussion and conclusion}

The data presented in our work are of considerable interest for reconstruction of solar radiation characteristics from the ionosphere observational data and for studying the ionization processes caused by solar ultraviolet radiation. In addition, our results are also important for the calibration and correction of IRI and NeQuick models by using GEC data.

Our data are in agreement with the convictions of some other authors (for example, Bilitza, 2001) about the use of UV solar radiation in addition to the generally accepted F10.7 index of solar activity for ionosphere modeling. Global electron content might be used as a new index of solar activity, since it reflects the changes in solar activity and correlates very well with solar UV radiation and solar radio emission at the $10.7 \mathrm{~cm}$ wavelength.

The absolute GEC values are of interest on their own as far as they are obtained within the simple and physical model of the refractive index of the ionosphere plasma (HofmannWellenhof et al., 1992). From this point of view experimen- tal GEC values are as important as maximum electron density values of $\mathrm{E}$ or $\mathrm{F} 2$ layers, which are determined from the measurements of the critical frequency at the ionosphere stations and used for reconstruction of solar radiation parameters (Beynon and Brown, 1959; Nusinov, 2004). Thus, it is possible to use GEC values, as well as maximum electron density values, for the calibration of parameters of solar UV radiation models, as well as of different ionosphere models.

Our results show that GEC very well reflects the dynamics of active formations on the Sun's surface during the solar cycle (Mordvinov and Plyusnina, 2001; Mordvinov and Willson, 2003). Figure 9 illustrates the qualitative explanation of this effect during periods of low (on the left) and high solar activity (on the right). The dependence of intensity of UV solar radiation $U(\lambda)$ (where $\lambda$ is the solar longitude) is shown in Fig. 9; it is transformed to a function of time $U(t)$ as the Sun rotates. A form of this curve depends on the number of active formations on the surface of the Sun, and its amplitude depends on solar activity level. 
During the falling and rising periods of solar activity, very few contrasting, long-living, powerful, active formations arise at the Sun, which can exist during several rotations of the Sun (Vitinsky et al., 1986; Mordvinov and Willson, 2003; Veselovsky et al., 2004). This causes deeper modulation of GEC with a period equal to the period of rotation of the Sun (Fig. 9, GEC(t) in the center). On the contrary, during high solar activity, much more active formations appear and move at the surface of the Sun. In this case the dependence $U(\lambda)$ has many maximal values. Therefore, despite the fact that the mean amplitude of the EUV flux increases at periods of maximal solar activity, the relative amplitude of the 27-day modulation decreases (Fig. 9, GEC(t) on the right). The least level of the 27-day modulation is observed at the period of the minimum of solar activity when the sunspot number also tends to zero (Fig. 9, GEC(t) on the left).

The above-mentioned mechanism of the 27-day modulation agrees with a character of the global solar asymmetry during extremely high solar activity during OctoberNovember 2003 (Sect. 5 and Fig. 9 in Veselovsky et al., 2004). The authors noted that "the brighter side of the Sun with powerful, active regions was facing the Earth from 19 October to 4 November. The central dates in this respect are 26-27 October. The darker side of the Sun with weaker magnetic fields was facing the Earth on 12 October and 11 November. Thus, the global asymmetry of the Sun clearly observed at the phase of growth and near the maximum of solar activity in the 23 rd cycle does exist at the declining phase."

This lag (of about 2 days) of the 27-day GEC variations, as compared to the corresponding changes in the EUV and radio 10.7-cm solar emissions (Figs. 7 and 8), can be caused by significantly greater time constants that characterize the thermosphere, as GEC variations are caused not only by changes in the solar ionizing radiation but also by the processes in the thermosphere (Ivanov-Kholodny and Nikolsky, 1969). Then, as solar radiation flux increases, thereby ionizing the ionosphere and heating the thermosphere, the temperature and total density of the atmosphere increase, and the speed and direction of the neutral wind change (Bryunelli and Namgaladze, 1988). It is also necessary to take into account that time scales of the electron content variations in the Earth's plasmasphere are changed within the range from 2 to 5 days (Krinberg and Taschilin, 1984). However, detailed analysis of the factors responsible for this lag of 27-day GEC variations relative to corresponding changes in the UV and F10.7 fluxes is out of the scope of this paper.

\footnotetext{
Acknowledgements. We acknowledge academician G. A. Zherebtsov, V. V. Pipin, V. G. Eselevich, A. V. Mordvinov, L. A. Plyusnina for their support and interest in our work and S. V. Voeykov, E. A. Kosogorov and I. N. Fedulina for their help in data preparing.

The authors thank the Center for Orbit Determination in Europe, University of Berne, Switzerland (CODG), Jet Propulsion Laboratory of California Institute of Technology (JPLG), Grup Universitat
}

Politecnica de Catalunya (UPCG), European Space Agency Group (ESAG) for the GIM available from ftp://cddisa.gsfc.nasa.gov/pub/ gps/products/ionex.

We are grateful to the Dominion Radio Astrophysical Observatory (DRAO, Canada) for the data of 10.7-cm radio emission, the Space Environment Technologies Company for the data of Rsn and Space Sciences Center of the USC for the data of solar UV flux.

The work was supported by the RFBR-GFEN grant N 06-0539026, RFBR grant 07-05-00127, the SB RAS collaboration project N 3.24 .

Topical Editor M. Pinnock thanks T. Tsugawa for his help in evaluating this paper.

\section{References}

Akasofu, S. I. and Chapmen, S.: Solar-Terrestrial Physics, 512 pp., Pergamon, Oxford, 1972.

Afraimovich, E. L., Astafyeva, E. I., and Zhivetiev, I. V.: Solar activity and global electron content, Doklady Earth Sciences, 409A, N6, 921-924, 2006a.

Afraimovich, E. L., Astafyeva, E. I., Oinats, A. V., Yasukevich, Yu. V., and Zhivetiev, I. V.: Global electron content as a new index of solar activity. Comparison with IRI modeling results, IRI news, 13(1), October 2006, A5, 2006b.

Afraimovich, E. L., Astafyeva, E. I., Oinats, A. V., Yasukevich, Yu. V., and Zhivetiev, I. V.: Global electron content and solar activity: comparison with IRI modeling results. Proceedings of IGS-2006, Darmstadt, http://nng.esoc.esa.de/ws2006/Papers/ p_Afraimovich_GEC_IRI_IGS_update.pdf.gz, 8-11 May 2006c.

Balan, N., Bailey, G. J., and Su, Y. Z.: Variations of the ionosphere and related solar fluxes during solar cycles 21 and 22, Adv. Space Res., 18(1), 11-14, 1996.

Beynon, W. J. G. and Brown, G. M.: Region E and solar activity, J. Atmos. Terr. Phys., 15, 168-174, 1959.

Bilitza, D.: International Reference Ionosphere 1990, NSSDC 9022, Greenbelt, Maryland, 1990.

Bilitza, D.: International reference ionosphere, Radio Sci., 36(2), 261-275, 2001.

Bryunelli, B. E. and Namgaladze, A. A.: Physics of the Ionosphere, 528 pp., Nauka, Moscow (in Russian), 1988.

Coisson, P., Radicella, S. M., and Nava, B.: Comparisons of experimental topside electron concentration profiles with IRI and NeQuick models, Annals of Geophysics, 45(1), 111-116, 2002.

Coisson, P. and Radicella, S. M.: The IRI topside parameters, Adv. Radio Sci., 2, 249-251, 2004, http://www.adv-radio-sci.net/2/249/2004/.

Coisson, P. and Radicella, S. M.: Ionospheric topside models compared with experimental electron density profiles, Annals of Geophysics, 48(3), 497-503, 2005.

Coisson, P., Radicella, S. M., Leitinger, R., and Nava, B.: New option for IRI topside electron density profile using NeQuick model, IRI News, 12(1, 2, 3), 5-7, 2005.

Giovanni, D. and Radicella, S. M.: An analytical model of the electron density profile in the ionosphere, Adv. Space Res., 10(11), 27-30, 1990.

Hofmann-Wellenhof, B., Litchtenegger, H., and Collins, J.: Global Positioning System: Theory and Practice, 327 pp., SpringerVerlag Wien, New York, 1992. 
Ivanov-Kholodny, G. S. and Nikolsky, G. M.: The sun and ionosphere, 456 pp, Nauka, Moscow (in Russian), 1969.

ITU-2004: Ionospheric propagation data and prediction methods required for the design of satellite services and systems, Recommendation ITU-R P.531-7, 2004.

Jakowski, N., Fichtelmann, B., and Jungstand, A.: Solar activity control of Ionospheric and thermospheric processes, J. Atmos. Terr. Phys., 53, 1125-1130, 1991.

Jakowski, N., Sardon, E., and Schluter, S.: GPS-based TEC observations in comparison with IRI95 and the european TEC model NTCM2, Adv. Space Res., 22, 803-806, 1998.

Jakowski, N., Heise, S., Wehrenpfennig, A., Schluter, S., and Reimer, B.: GPS/GLONASS-based TEC measurements as a contributor for space weather, J. Atm. Terr. Phys., 64(5-6), 729-735, 2002.

Judge, D. L., McMullin, D. R., Ogawa, H. S., et al.: First Solar EUV Irradiances Obtained from SOHO by the Celias/Sem., Solar Phys., 177(1/2), 161-173, 1998.

Kane, R. P., de Paula, E. R., and Triverdi, N. B.: Variations of solar EUV, UV and ionospheric $\mathrm{f}_{0} \mathrm{~F} 2$ related to the solar rotation period, Ann. Geophys., 13, 717-723, 1995, http://www.ann-geophys.net/13/717/1995/.

Krinberg, I. A. and Taschilin, A. V.: Ionosphere and Plasmosphere, 188 pp., Nauka, Moscow (in Russian), 1984.

Kumar, P. and Foufoula-Georgiou, E.: Wavelet Applications in Geophysics: A Review, Rev. Geophys., 35, 385-412, 1997.

Leitinger, R., Zhang, M., and Radicella, S. M.: An improved bottomside for the ionospheric electron density model NeQuick, Ann. Geophys., 48(3), 525-534, 2005.

Liu, L., Wan, W., Ning, B., Pirog, O. M., and Kurkin, V. I.: Solar activity variations of the ionospheric peak electron density, J. Geophys. Res., Ill, A08304, doi:10.1029/2006JA011598, 2006.
Mannucci, A. J., Wilson, B. D., Yuan, D. N., Ho, C. M., Lindqwister, U. J., and Runge, T. F.: A global mapping technique for GPSderived ionospheric TEC measurements, Radio Sci., 33(3), 565582, 1998.

Mordvinov, A. V. and Plyusnina, L. A.: Coherent structures in the dynamics of the large-scale solar magnetic field, Astronomy reports, 45, 652-658, 2001.

Mordvinov, A. V. and Willson, R. C.: Effect of large-scale magnetic fields on total solar irradiance, Solar Phys., 215, 5-16, 2003.

Nusinov, A. A. and Katyushina, V. V.: Lyman-alpha line intensity as a solar activity index in the far ultraviolet range, Solar Phys., 152, 201-206, 1994.

Nusinov, A. A.: The Ionosphere as a Natural Detector for Studying Long-Period Variations in the Fluxes of Solar Geoeffective Radiation, Geomagnetism I Aeronomia, 44(6), 718-725, 2004.

Oyama, K.-I., Noguchi, K., Izawa, M., Saito, A., Otsuka, Y., and Tohyama, F.: Local time, annual, latitude, and seasonal variations of total electron content over Japan, Institute of space and astronautical science, ISAS Research Note, 796, 2005.

Radicella, S. M. and Leitinger, R.: The evolution of the DGR approach to model electron density profiles, Adv. Space Res., 27(1), 35-40, 2001.

Schaer, S., Beutler, G., and Rothacher, M.: Mapping and predicting the ionosphere, Proceedings of the IGS AC Workshop, Darmstadt, Germany, 9-11 February, 307-320, 1998.

Veselovsky, I. S., Panasyuk, M. I., Avdyushin, S. I., Bazilevskaya, G. I., et al.: Solar and Heliospheric Phenomena in OctoberNovember 2003; Causes and Effects, Cosmic Res., 42(5), 435488, 2004.

Vitinsky, Yu. I., Kopecky, M., and Kuklin, G. V.: Statistics of sunspot activity, pp. 296, Nauka, Moscow (in Russian), 1986. 